Palavras chave: Área ripária Floresta Ombrófila Mista Montana Análise multivariada

Histórico:

Recebido 27/06/2013

Aceito 17/09/2015

Keywords:

Riparian area

Montane Mixed Ombrophilous Forest Multivariate analysis

Correspondência: dannirorato@hotmail.com
Daniele Guarienti Rorato', Maristela Machado Araujo', Luciane Almeri Tabaldi', Thaíse da Silva Tonetto', Ana Paula Moreira Rovedder', Adriana Falcão Dutra'

\section{INFLUÊNCIA DOS FATORES AMBIENTAIS NO COMPONENTE ARBÓREO DE FRAGMENTOS FLORESTAIS EM SÃO FRANCISCO DE PAULA - RIO GRANDE DO SUL}

RESUMO: O presente trabalho teve como objetivo geral caracterizar a influência do solo e da topografia no componente arbóreo de fragmentos, no entorno do Reservatório Divisa, na região dos Campos de Cima da Serra, Sul do Brasil. O levantamento da vegetação foi realizado em quatro fragmentos, sendo em cada parcela $(10 \times 20 \mathrm{~m})$ efetuada a identificação e medição da circunferência à altura do peito (CAP) dos indivíduos com CAP $\geq 30 \mathrm{~cm}$. Os dados de vegetação foram submetidos à análise de agrupamento. Adicionalmente, foram obtidas as variáveis ambientais, como declividade média das parcelas e amostras de solo, na camada de $0-20 \mathrm{~cm}$ de profundidade. A correlação entre os dados ambientais e de vegetação foi realizada por meio da Análise de Correspondência Canônica. As características físicas do solo não apresentaram influência nos grupos formados e nas espécies. Nos capões, a constante presença do gado influenciou negativamente a estrutura do sub-bosque, assim como as características químicas do solo. Alumínio e saturação por alumínio apresentaram maior influência nas espécies predominantes da mata ciliar enquanto cobre e enxofre influenciaram as espécies presentes nos capões. Espécies como Araucaria angustifolia, Eugenia uruguayensis, Blepharocalyx salicifolius, Calyptranthes concinna, Lithraea brasiliensis, Myrsine coriacea, Ocotea pulchella e Sebastiania commersoniana são adaptadas à mata ciliar, podendo ser utilizadas na recuperação e enriquecimento desses ambientes.

\section{INFLUENCE OF ENVIRONMENTAL FACTORS IN TREE COMPONENT OF FOREST FRAGMENTS IN SÃO FRANCISCO DE PAULA - RIO GRANDE DO SUL}

ABSTRACT: This study aimed to characterize the influence of the soil and topography in the tree component of the fragments, in around the Divisa Reservoir, in the region of the Campos de Cima da Serra, Southern Brazil. The vegetation survey was conducted in four fragments, with each plot $(10 \times 20 \mathrm{~m})$ performed the identification and measurement of the circumference at breast height (CAP) of subjects with CAP $\geq 30 \mathrm{~cm}$. The vegetation data were subjected to cluster analysis. In addition, environmental variables were obtained as an average slope of plots and soil samples at $0-20 \mathrm{~cm}$ depth. The correlation between vegetation data and environmental data was performed by means of Canonical Correspondence Analysis. The soil physical characteristics showed no influence on the groups formed and species. In the geldings, the constant presence of cattle influenced negatively the structure of the understory, as well as the chemical characteristics of the soil. Aluminium and aluminum saturation have higher influenced on the predominant species of riparian vegetation as influenced copper and sulfur species present in geldings. Species such as Araucaria angustifolia, Eugenia uruguayensis, Blepharocalyx salicifolius, Calyptranthes concinna, Lithraea brasiliensis, Myrsine coriacea, Ocotea pulchella and Sebastiania commersoniana are adapted to riparian and can be used in the recovery and enrichment of these environments. 


\section{INTRODUÇÃO}

A Floresta Ombrófila Mista Montana (FOM Montana) ou Floresta de Araucária ocorre entre 400 e $1000 \mathrm{~m}$ de altitude (IBGE, 20I2), e caracteriza-se pela mistura de floras, na qual Araucaria angustifolia (Bertol.) Kuntze, espécie típica dessa fitofisionomia forma uma cobertura emergente característica. Os Campos de Cima da Serra ou Campos de Altitude formam, com frequência, mosaicos com essa formação, caracterizando um sistema de transição campo-floresta fortemente influenciado pelas atividades humanas (PILLAR et al., 2009). Essas áreas de transição, assim como a FOM Montana vêm sendo substituídas pela expansão da agricultura, pecuária, silvicultura de espécies exóticas e também pela construção de obras de utilidade pública, acarretando perda da biodiversidade local (LEITE, 2002; BOND-BUCKUP, 2008).

Sendo a floresta um ambiente com grande variação na composição florística, riqueza de espécies e estrutura, a descrição, classificação e ordenação da vegetação correlacionada a fatores ambientais, fornece informações importantes sobre o ambiente em estudo. Conforme Felfili e Rezende (2003) a formação de agrupamentos na vegetação está relacionada às condições ambientais associadas, que favorecem a ocorrência, ou não, de determinadas espécies. No mesmo intuito, métodos de ordenação são empregados para determinar as relações existentes entre a vegetação e o ambiente (MATTEUCCI; COLMA, 1982). Dentre esses métodos, destaca-se a Análise de Correspondência Canônica (CCA), a qual permite a ordenação conjunta e simultânea das espécies com as variáveis ambientais, cujo resultado expressa a variabilidade dos dados (KENT; COKER, 1992).

Assim, correlacionar informações da vegetação com variáveis ambientais, permite inferir sobre a conservação biológica desses ecossistemas e estratégias para sua recuperação. Nesse sentido, foi desenvolvido um estudo em fragmentos de Floresta Ombrófila Mista Montana, no entorno do Reservatório Divisa, São Francisco de Paula, RS, visando: a) avaliar a influência de atributos do solo na estrutura do componente arbóreo dos fragmentos; b) determinar a influência de variáveis do solo sobre a distribuição espacial das espécies predominantes, com ênfase nas de mata ciliar; e c) identificar espécies que apresentam potencial para recuperação e enriquecimento de mata ciliar nos domínios da Floresta Ombrófila Mista Montana.

\section{MATERIAL E MÉTODOS}

A área de estudo localiza-se no entorno do Reservatório Divisa, município de São Francisco de Paula, RS (29 26' 52" S de latitude e 50 35' 02" W de longitude), com altitude média de $907 \mathrm{~m}$. O clima da região é subtropical do tipo $\mathrm{Cfb}$, cuja temperatura média do mês mais quente é inferior a $22^{\circ} \mathrm{C}$ e do mês mais frio superior a $3^{\circ} \mathrm{C}$ (MORENO, 196I), com precipitação média anual de $2.500 \mathrm{~mm}$ (NIMER, 1990).

A região é denominada como Campos de Cima da Serra, no qual os campos formam mosaicos com os fragmentos de Floresta Ombrófila Mista Montana (IBGE, 20I2). Os solos dominantes na região foram classificados como Cambissolos Húmicos Alumínicos típicos, Cambissolos Háplicos Alumínicos organossólicos, Neossolos Regolíticos Húmicos lépticos e Neossolos Litólicos Húmicos típicos (STRECK et al., 2008). Tonetto (20II) observou a presença de Neossolos Litólicos, classificados até o segundo nível categórico, nos fragmentos florestais analisados nesse estudo.

O levantamento da vegetação foi realizado em quatro fragmentos florestais no entorno do Reservatório Divisa, denominados de Fragmento I (FI - 9 ha), Fragmento 2 (F2 - 9 ha), Fragmento 4 (F4 - 2 ha) e Fragmento 5 (F5 - 9 ha), totalizando 57 parcelas inventariadas. As parcelas $(10 \times 20 \mathrm{~m})$ foram sistematicamente distribuídas em faixas paralelas nos fragmentos, as quais podem ser observadas em Rorato (20/2). Nessas parcelas foi realizada a identificação e a medição, com fita métrica, dos indivíduos com circunferência à altura do peito $(\mathrm{CAP}) \geq 30 \mathrm{~cm}$. Com base nesses dados, foram excluídas espécies raras, com menos de 5 indivíduos amostrados, obtendo-se dois grupos (GRI - Mata Ciliar e GR2 - Remanescente de Floresta Ombrófila Mista Montana, comumente denominado de capão) pelo método TWINSPAN (TWoway INdicator SPecies ANalysis) (GAUCH, 1982), no programa PC-ORD ${ }^{T M}$ for Windows versão 5.10 (McCUNE; MEFFORD, 2006).

Adicionalmente ao levantamento da vegetação foi obtida a variável declividade nas parcelas inventariadas, com uso de clinômetro Suunto ${ }^{\circledR}$, expressando assim a variação do gradiente topográfico entre as parcelas, dentro de cada faixa. Para a caracterização química e física do solo, foram coletadas três amostras simples de solo, na camada 0 a $20 \mathrm{~cm}$, formando a amostra composta, em cada uma das 32 parcelas inventariadas. Foram realizadas análises químicas, conforme metodologia proposta por Tedesco et al. (1995), sendo determinados $\mathrm{pH}$ em água e 
em $\mathrm{KCl}, \mathrm{V} \%$ (saturação por bases), m\% (saturação por alumínio), $\mathrm{H}+\mathrm{Al}$ (acidez potencial), CTC efetiva, CTC $\mathrm{pH}$, além dos conteúdos de matéria orgânica (MO), alumínio livre $\left(\mathrm{Al}^{+3}\right)$, cálcio $\left(\mathrm{Ca}^{+2}\right)$, magnésio $\left(\mathrm{Mg}^{+2}\right)$, potássio $\left(\mathrm{K}^{+}\right)$, enxofre $\left(\mathrm{S}^{-2}\right)$, fósforo $\left(\mathrm{P}^{-3}\right)$, cobre $\left(\mathrm{Cu}^{+2}\right)$, boro $\left(\mathrm{B}^{+3}\right)$ e zinco $\left(\mathrm{Zn}^{+2}\right)$.

Como variáveis físicas foram determinadas a granulometria (percentual de areia grossa, areia fina, silte e argila), pelo Método da Pipeta (GEE; BAUDER, 1986), a umidade gravimétrica e densidade aparente do solo, obtidas por meio da utilização de Anel de Koppek (EMBRAPA, 1997), e calculada conforme Reichardt e Timm (2004).

A correlação entre os dados de vegetação e dados ambientais foi realizada por meio da Análise de Correspondência Canônica (CCA), com uso do programa PC-ORD ${ }^{T M}$ for Windows versão 5.10 (McCUNE; MEFFORD, 2006), visando à ordenação entre variáveis de diferentes naturezas (NAPPO et al., 2000; ARAUJO et al., 20I0; SANTOS et al., 20I2). Assim, os dados de entrada consistiram em uma matriz principal de densidade das espécies (indivíduos/parcela), com CAP $\geq 30 \mathrm{~cm}$, composta por 32 parcelas (linhas) e 22 espécies (colunas), e uma matriz secundária com 32 parcelas (linhas) e 24 variáveis ambientais (colunas). Nessa análise, foram desprezadas as espécies presentes com densidade inferior a 5 indivíduos por parcela, e os dados transformados visando atenuar os efeitos dos desvios provocados por valores muito elevados aplicando In $(a+I)$, sendo "a" o número de indivíduos em cada parcela (PALMER, 1993; CARVALHO et al., 2005).

Com base na análise preliminar, foram removidas variáveis fracamente correlacionadas, considerando valor de correlação inferior a 0,4. Após, os dados foram novamente processados com cinco variáveis ambientais $\left(\mathrm{Al}^{+3}, \mathrm{Ca}^{+2}, \mathrm{~S}^{-2}, \mathrm{Cu}^{+2}\right.$ e $\left.\mathrm{m} \%\right)$, com significância das correlações entre as matrizes analisadas pelo teste de permutação de Monte Carlo, utilizando 5000 interações (McCUNE; GRACE, 2002).

A interpretação dos teores nutricionais foi realizada de acordo com a Sociedade Brasileira de Ciência do Solo/Comissão de Química e Fertilidade do Solo (SBCS/CQFS 2004), que apesar de ser referencial para a agricultura e silvicultura de espécies comerciais, foi utilizada para inferir sobre as espécies florestais nativas, ainda pouco estudadas quanto a esse aspecto.

\section{RESULTADOS E DISCUSSÃO}

As características físicas analisadas no solo desse estudo (Neossolo Litólico) além das outras 12 variáveis químicas avaliadas e a declividade não apresentaram correlação com os grupos fitossociológicos nem com as espécies (autovalores <0.4).

Em ambos os grupos (GRI (Mata Ciliar) e GR2 (Remanescente de Floresta Ombrófila Mista Montana), pode-se observar os elevados valores de $\mathrm{H}+\mathrm{Al}$ e $\mathrm{Al}^{+3}$, devido às altas concentrações de $\mathrm{MO}$, refletindo no $\mathrm{pH}$ ácido (Tabela I). Nessa situação, devido a $\mathrm{MO}$ estar na superfície do solo, em função da sua lenta decomposição, decorrente das menores temperaturas nessa região, comparativamente às outras do $\mathrm{RS}$, os cátions $\left(\mathrm{H}^{+}\right.$e $\left.\mathrm{Al}^{+3}\right)$ não se encontram aderidos às cargas negativas do solo (SILVA et al., 2006). Isso é confirmado por Lepsch et al. ( 1982 ), os quais relataram que a taxa de decomposição da matéria orgânica varia em função das condições de TABELA I Valores médios relativos aos atributos químicos do solo e interpretação, conforme a SBCS/ CQFS (2004) para os grupos fitossociológicos, Reservatório Divisa, São Francisco de Paula, RS.

TABLE I Mean values for the soil chemical properties and interpretation, as SBCS/CQFS (2004) for groups phytosociological, Divisa Reservoir, São Francisco de Paula, RS.

\begin{tabular}{|c|c|c|c|c|}
\hline \multirow[b]{2}{*}{ Atributos Químicos } & \multicolumn{4}{|c|}{ Grupos } \\
\hline & GRI & Interpretação & GR2 & Interpretação \\
\hline Acidez ativa ( $\mathrm{pH}$ água) & 4,7 & Baixo & 4,8 & Baixo \\
\hline $\begin{array}{l}\text { Acidez potencial } \\
(\mathrm{H}+\mathrm{Al})\left(\mathrm{cmol}_{\mathrm{c}} \cdot \mathrm{dm}^{-3}\right)\end{array}$ & 21,7 & - & 19,1 & - \\
\hline $\mathrm{Al}^{+3}\left(\mathrm{cmol}_{\mathrm{c}} \cdot \mathrm{dm}^{-3}\right)$ & 4,9 & - & 3,1 & - \\
\hline $\mathrm{Ca}^{+2}\left(\mathrm{cmolc} \cdot \mathrm{dm}^{-3}\right)$ & 3,0 & Médio & 5,8 & Alto \\
\hline $\mathrm{Mg}^{+2}\left(\mathrm{cmolc} \cdot \mathrm{dm}^{-3}\right)$ & $\mathrm{I}, \mathrm{I}$ & Alto & $\mathrm{I}, 5$ & Alto \\
\hline $\mathrm{K}^{+}\left(\mathrm{mg} \cdot \mathrm{dm}^{-3}\right)$ & 0,2 & Muito Baixo & 0,3 & Muito Baixo \\
\hline $\mathrm{P}^{-3}\left(\mathrm{mg} \cdot \mathrm{dm}^{-3}\right)$ & 3,7 & Muito Baixo & 2,5 & Muito Baixo \\
\hline $\mathrm{S}^{-2}\left(\mathrm{mg} \cdot \mathrm{dm}^{-3}\right)$ & 10,0 & Alto & 14,8 & Alto \\
\hline $\mathrm{Cu}^{+2}\left(\mathrm{mg} \cdot \mathrm{dm}^{-3}\right)$ & 2,8 & Alto & 6,5 & Alto \\
\hline $\mathrm{Zn}^{+2}\left(\mathrm{mg} \cdot \mathrm{dm}^{-3}\right)$ & 2,6 & Alto & 4,1 & Alto \\
\hline $\mathrm{B}^{+3}\left(\mathrm{mg} \cdot \mathrm{dm}^{-3}\right)$ & 0,5 & Alto & 0,5 & Alto \\
\hline $\mathrm{MO}(\%)$ & 6,4 & Alto & 5,9 & Alto \\
\hline $\mathrm{m} \%$ & 56,9 & Baixo & 34,2 & Médio \\
\hline V\% & 19,9 & Muito Baixo & 34,0 & Muito Baixo \\
\hline CTC efet $\left(\mathrm{cmol}_{\mathrm{c}} \cdot \mathrm{m}^{-3}\right)$ & 9,2 & Baixo & 10,7 & Médio \\
\hline CTC pH7 (cmolc $\left.\cdot \mathrm{dm}^{-3}\right)$ & 26,0 & Muito Baixo & 26,0 & Muito Baixo \\
\hline
\end{tabular}

Sendo: Fósforo extraído pelo método Mehlich; CTC efet: capacidade de troca de cátions efetiva; CTC pH7: capacidade de troca de cátions potencial; GRI: Grupol (Mata ciliar); GR2: Grupo 2 (Remanescente de Floresta Ombrófila Mista Montana). 
temperatura, umidade, relevo, material de origem, entre outros.

Além disso, conforme Indrasumunar et al. (20I2), essa acidez do solo pode acarretar deficiência de nutrientes minerais, principalmente cálcio, magnésio, fósforo e molibdênio limitando assim, o crescimento das plantas. Assim, supõe-se que as espécies predominantes nos locais estudados apresentam eficiência no uso desses nutrientes.

De forma semelhante, a variação na concentração dos macronutrientes $\left(\mathrm{Ca}^{+2}, \mathrm{Mg}^{+2}, \mathrm{~K}^{+}, \mathrm{P}^{-3}\right.$

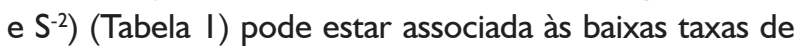
ciclagem de nutrientes em regiões de clima frio, como a estudada. Nesses locais, grande parte da $\mathrm{MO}$ e dos nutrientes permanece no solo e em sedimentos, ao invés de ser reciclada dentro das estruturas orgânicas do sistema (ODUM, 1988).

No caso dos micronutrientes $\left(\mathrm{Cu}^{+2}, \mathrm{Zn}^{+2}\right.$ e $\left.\mathrm{B}^{+3}\right)$ (Tabela I), as elevadas concentrações são características dos solos da região, desenvolvidos a partir de rochas efusivas básicas, intermediárias e ácidas (basalto, riodacitos ou riolitos, respectivamente), os quais apresentam baixa fertilidade natural (MOSER, 1990).

A análise da distribuição das parcelas e espécies, com relação aos fatores ambientais $\left(\mathrm{Al}^{+3}, \mathrm{Ca}^{+2}, \mathrm{~S}^{-2}\right.$, $\mathrm{Cu}^{+2}$ e $\mathrm{m} \%$ ), por meio da Análise de Correspondência Canônica, para a vegetação arbórea (CAP $\geq 30 \mathrm{~cm}$ ), indicou autovalores de 0,209 e 0,067, para o $1^{\circ}$ e $2^{\circ}$ eixos, respectivamente. Conforme Carvalho et al. (2005), autovalores baixos indicam que a maior parte das espécies encontram-se distribuídas em toda a área, variando apenas em sua abundância.

A porcentagem acumulada de variância explicada foi de $16,3 \%$, sendo I2,3\% explicado pelo eixo I e 4,0\%, no eixo 2. Com base nesses valores, pode-se considerar que as variáveis ambientais de solo analisadas explicam, parcialmente, as variações existentes na distribuição das espécies. Desse modo, a variação restante, não explicada pelas variáveis ambientais utilizadas nesse estudo, pode estar associada àquelas não registradas, como por exemplo, luminosidade, pedregosidade, profundidade efetiva do perfil do solo, entre outros, os quais devem ser considerados em estudos futuros realizados em Neossolos Litólicos, pois podem exercer efeito sobre a organização da comunidade. Resultados semelhantes foram encontrados por Nappo et al. (2000), que obtiveram explicação semelhante com as variáveis ambientais consideradas.

$\mathrm{Na}$ Figura I verifica-se que as variáveis $\mathrm{S}^{-2}$ e $\mathrm{Cu}^{+2}$ apresentaram correlação com o Grupo 2 (GR2), ambiente caracterizado como um remanescente de Floresta Ombrófila Mista Montana, comumente denominado de capão. Os capões encontram-se sob influência do uso intensivo dos campos na pecuária. Os dejetos produzidos pelo gado, os quais podem conter elevadas concentrações desses elementos (DA VEIGA et al., 20I2), juntamente com a deposição da biomassa sob o dossel da floresta, compõem a matéria orgânica do solo, contribuindo para a situação evidenciada.

Observa-se que apesar de $\mathrm{S}^{-2}$ e $\mathrm{Cu}^{+2}$ apresentarem altos teores para ambos os grupos (Tabela I), os maiores valores no GR2 influenciaram a classificação desse grupo (Figura I). $\mathrm{O}$ fato de $\circ \mathrm{S}^{-2}$ ter, entre as principais fontes, resíduos orgânicos vegetais e animais (ALVAREZ et al., 2007), permite inferir que a presença do gado no GR2, além de alterar a estrutura do sub-bosque, conforme evidenciado em Rorato (2012), influencia nas características químicas do solo.

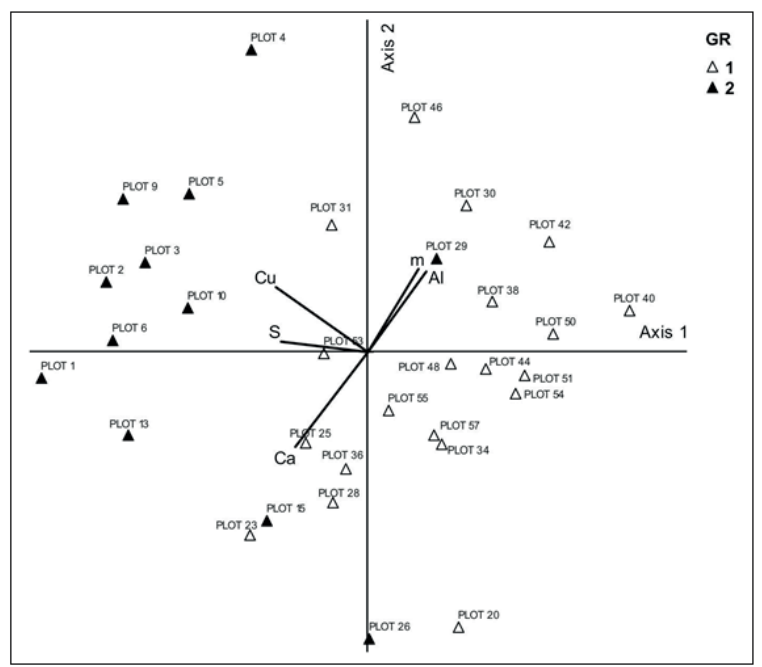

Figura I Diagrama de ordenação dos grupos analisados com as variáveis ambientais para os dois primeiros eixos produzidos pela Análise de Correspondência Canônica, Reservatório Divisa, São Francisco de Paula, RS. Sendo GR: Grupo; Plot: Parcelas; Axis: Eixos.

Figure I Diagram of sorting the groups analyzed environmental variables for the first two axes produced by Canonical Correspondence Analysis, Divisa Reservoir, São Francisco de Paula, RS. Being GR: Group; Plot: Plots; Axis: Axis.

Para esse mesmo grupo, o $\mathrm{Cu}^{+2}$ apresentou valor médio de $6,5 \mathrm{mg} \cdot \mathrm{dm}^{-3}$, superior ao observado no GRI (2,8 $\mathrm{mg} \cdot \mathrm{dm}^{-3}$ ) (Tabela I). Níveis elevados de $\mathrm{Cu}^{+2}$ são tóxicos para as plantas e interferem em importantes processos bioquímicos e fisiológicos tais como fotossíntese, respiração e absorção de nutrientes (YRUELA, 2009). No entanto, Silva et al. (20ll), observaram que 
algumas espécies florestais apresentaram tolerância aos elevados teores de $\mathrm{Cu}^{+2}$ no solo, o que sugere a utilização dessas na revegetação de ambientes com elevados teores desses elementos $\left(\mathrm{S}^{-2}\right.$ e $\left.\mathrm{Cu}^{+2}\right)$.

Por outro lado, o $\mathrm{Al}^{+3}$ e $\mathrm{m} \%$ estão altamente correlacionados com o GRI, que representa a situação de mata ciliar, apesar de também estar fortemente correlacionado com uma parcela do GR2 (Plot 29) (Figura I). Embora classificada como GR2, essa parcela representa a borda da mata ciliar, adjacente ao capão, justificando sua localização no diagrama.

A ação da matéria orgânica aparece influenciando os grupos com os elevados teores de $\mathrm{Al}^{+3}$ livre na solução do solo, devido ao maior teor $\mathrm{MO} \%$, em função das lentas taxas de intemperismo na região, condicionadas pela altitude, clima frio, material de origem basáltico e relevo (SILVA et al., 2006). Todos esses aspectos, associados à maior umidade, que além de ser própria da região, é potencializada na mata ciliar (GRI), acarreta menor saída do $\mathrm{Al}^{+3}$ do complexo do solo. Esse resultado pode ser confirmado ao analisar os valores médios de $\mathrm{Al}^{+3}$ no GRI $\left(4,9 \mathrm{cmol}_{\mathrm{c}} \cdot \mathrm{dm}^{-3}\right)$ em comparação ao GR2 $\left(3, \mathrm{I} \mathrm{cmol}_{\mathrm{c}} \cdot \mathrm{dm}^{-3}\right)($ Tabela I).

A saturação por alumínio ( $\mathrm{m} \%$ ) apresentou valores de $56,9 \%$ (GRI) e 34,2\% (GR2). Os efeitos do $\mathrm{Al}^{+3}$ em concentrações tóxicas manifestam-se na parte aérea e radicular, por meio de sintomas anatômicos, morfológicos e redução no crescimento, devido à diminuição no alongamento das raízes e a consequente redução na possibilidade de absorção de nutrientes (HIRANO et al., 2003). Desse modo, as elevadas concentrações de $\mathrm{Al}^{+3}$ e $\mathrm{Cu}^{+2}$, podem ter influenciado de forma negativa o desenvolvimento e expansão das florestas no local, merecendo estudos para as espécies florestais nativas.

O $\mathrm{Ca}^{+2}$ foi a variável que melhor se correlacionou com ambos os grupos formados. Esse nutriente possui a função de regulação da hidratação, ativação de enzimas e regulador do crescimento, sendo acumulado, principalmente, nas folhas e na casca das plantas (LARCHER, 2000).

Tendo em vista que o comprimento da seta no biplot é proporcional à magnitude de mudança na direção indicada (KENT; COKER, 1992), observou-se a menor influência das variáveis ambientais sobre as espécies do que em relação às parcelas (grupos), o que não permite inferir sobre possíveis exigências nutricionais das espécies. Apesar disso, observou-se que Eugenia uruguayensis Cambess. e Myrsine coriacea (Sw.) R.Br., ambas espécies indicadoras do GRI (RORATO, 2012), apresentaram correlação com o aumento do $\mathrm{Al}^{+3}$ e com a m\% (FIGURA 2).

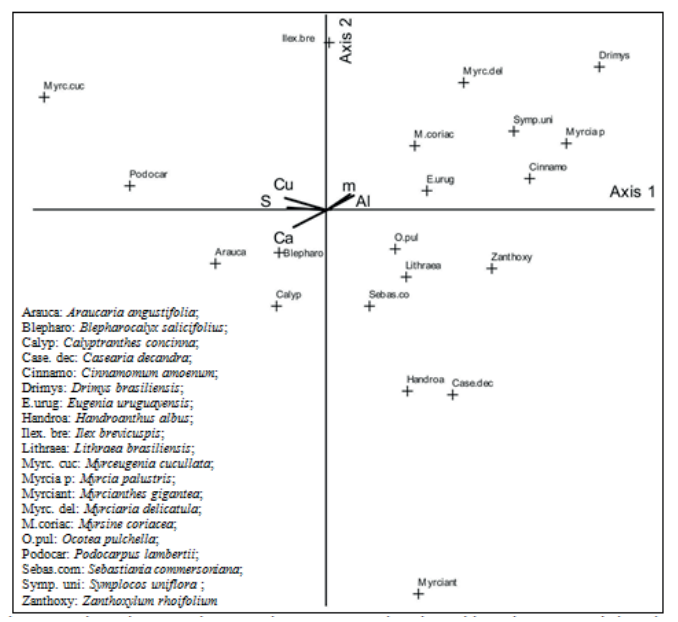

Figura 2 Diagrama de ordenação das espécies com as variáveis ambientais para os dois primeiros eixos produzidos pela Análise de Correspondência Canônica, Reservatório Divisa, São Francisco de Paula, RS.

Figure 2 Diagram of sorting the species analyzed environmental variables for the first two axes produced by Canonical Correspondence Analysis, Divisa Reservoir, São Francisco de Paula, RS.

Araucaria angustifolia, Blepharocalyx salicifolius (Kunth) O. Berg e Calyptranthes concinna DC. apresentaram maior correlação com a variável $\mathrm{Ca}^{+2}$. Essas espécies estão representadas proporcionalmente em ambos os grupos. No caso da A. angustifolia, a casca desprende-se em lâmina da parte superior do fuste, aumentando, assim, a deposição desse material no solo e, consequentemente, os teores do $\mathrm{Ca}^{+2}$ no sítio. Considerando o médio e alto teor desse elemento (Tabela I), respectivamente, no GRI e GR2, possivelmente, $B$. salicifolius e $C$. concinna, além de $A$. angustifolia, estão melhor correlacionadas a esse elemento. Dessa forma, essas espécies também podem ser observadas como potenciais para utilização em programas de recuperação de áreas alteradas, especialmente em ambiente ciliar.

Myrceugenia cucullata D. Legrand e, principalmente, Podocarpus lambertii Klotzsch, apresentam-se melhor correlacionadas com S $\mathrm{S}^{-2}$ e $\mathrm{Cu}^{+2}$. Verifica-se que esse grupo apresentou os maiores teores de $\mathrm{S}^{-2}$ e $\mathrm{Cu}^{+2}\left(14,8 \mathrm{e} 6,5 \mathrm{mg} \cdot \mathrm{dm}^{-3}\right)$, quando comparado ao GRI (10,0 e 2,8 $\left.\mathrm{mg} \cdot \mathrm{dm}^{-3}\right)$ (Tabela I), justificando a influência dessas variáveis sobre as duas espécies mencionadas. Além disso, apresentam menor potencial para uso na mata ciliar, considerando reduzida representatividade nesse ambiente, quando comparado ao GR2 (RORATO, 20I2). 
Sebastiania commersoniana L.B. Sm. \&. Downs, Lithraea brasiliensis March. e Ocotea pulchella (Nees) Mez, podem ser enfatizadas na mata ciliar, dada sua maior densidade (indivíduos.ha-1) (RORATO, 20I2), e ocorrência em solos com menores teores de $\mathrm{Cu}^{+2}$ e S$^{-2}$. Isso pode ser explicado, conforme Kent e Coker (1992), pois a linha que indica a variável explicativa (ambiental) pode ser estendida, no sentido contrário de sua origem, indicando que a menor influência da variável favorece os elementos do lado oposto.

\section{CONCLUSÃO}

As característicasfísicas dosolonãoapresentaram influência nos grupos formados e nas espécies. Alumínio e saturação por alumínio apresentaram maior influência nas espécies predominantes da mata ciliar enquanto cobre e enxofre influenciaram àquelas presentes nos capões. $A$. angustifolia, E. uruguayensis, B. salicifolius, C. concinna, $L$. brasiliensis, $M$. coriacea, $O$. pulchella e $S$. commersoniana podem ser testadas para a composição de arranjos na recuperação e enriquecimento de ambiente ciliar, nos domínios da Floresta Ombrófila Mista Montana.

\section{AGRADECIMENTOS}

A ANEEL e a CEEE por meio do Projeto "Fitossociologia de espécies nativas de mata ciliar na Barragem do Divisa", pelo auxilio logístico e financeiro.

\section{REFERÊNCIAS BIBLIOGRÁFICAS}

ALVAREZ, V. H.; ROSCOE, R.; KURIHARA, C. H. PEREIRA, N. F. Enxofre. In: NOVAIS, R.F. et al. Fertilidade do solo. Viçosa: Sociedade Brasileira de Ciência do Solo, 2007. Cap. 10, p. 595-644.

ARAUJO, M. M.; CHAMI, L.; LONGHI, S. L.; AVILA, A. L.; BRENA, D. A. Análise de agrupamento em remanescente de Floresta Ombrófila Mista. Ciência Florestal, Santa Maria, v. 20, n. I, p. I-I8. 2010.

BOND-BUCKUP, G. Biodiversidade dos Campos de Cima da Serra. Porto Alegre: Libretos, 2008. 146 p.

CARVALHO, D. A. et al. Distribuição de espécies arbóreoarbustivas ao longo de um gradiente de solos e topografia em um trecho de floresta ripária do Rio São Francisco em Três Marias, MG, Brasil. Revista Brasileira de Botânica, v. 28, n. 2, p. 329-345, 2005.

DA VEIGA, M.; PANDOLFO, C. M.; BALBINOT JUNIOR, A. A. Cobre e zinco no solo e no tecido vegetal após nove anos de uso de fontes de nutrientes associadas a sistemas de manejo em um Nitossolo Vermelho. Revista Agropecuária Catarinense, v. 25, n. 2, p. 79-84, 2012.
EMBRAPA - EMPRESA BRASILEIRA DE PESQUISA AGROPECUÁRIA. Manual de métodos de análises de solo. 2. ed. Rio de Janeiro: Ministério da Agricultura e do Abastecimento, 1997. 212p.

FELFILI, J. M.; REZENDE, R. P. Conceitos e métodos em fitossociologia. Comunicações Técnicas Florestais, Brasília, v. 5, n. I, 2003. 68p.

GAUCH, H. G. Multivariate analysis in community ecology. Cambridge: Cambridge University Press, 1982. 180p.

GEE, G. W.; BAUDER, J. W. Particle size analysis. In: KLUTE, A., ed. Methods of Soil Analysis: part I. 2. ed. Madison, American Society of Agronomy, 1986. p. 383-4I I.

HIRANO, Y.; ISOMURA, A.; KANEKO, S. Root morphology and nutritional status of Japanese red cedar saplings subjected to in situ levels of aluminum in forest soil solution. Journal of Forest Research, v. 8, p. 209-214, 2003.

IBGE - INSTITUTO BRASILEIRO DE GEOGRAFIA E ESTATÍSTICA. Manual técnico da vegetação brasileira: sistema fitogeográfico, inventário das formações florestais e campestres, técnicas e manejo de coleções botânicas, procedimentos para mapeamentos. 2a ed. Rio de Janeiro, 20I2, 275p.

INDRASUMUNAR, A.; MENZIES, N. W.; DART, P. J. Laboratory prescreening of Bradyrhizobium japonicum for low $\mathrm{pH}, \mathrm{Al}$ and Mn tolerance can be used to predict their survival in acid soils. Soil Biology \& Biochemistry, v. 48, p. I35- |4I, 2012.

KENT, M.; COKER, P. Vegetation description analyses. London: Behaven Press, 1992. 363p.

LARCHER, W. Ecofisiologia vegetal. São Carlos: Rima, 2000. 53 I p.

LEITE, P. F. Contribuição ao conhecimento fitoecológico do Sul do Brasil. Ciência \& Ambiente, Santa Maria, v. I, n. I, p. 5I-73. 2002.

LEPSCH, I. F.; SILVA, N. M.; ESPIRONELO, A. Relação entre matéria orgânica e textura de solos sob cultivo de algodão e cana-de-açúcar, no Estado de São Paulo. Bragantia, v. 4 I, p. 23 I-236, 1982.

MATTEUCCI, S. D.; COLMA, A. Metodologia para el estudio de la vegetacion. Washington: Secretaria General de la Organización de los Estados Americanos Programa Regional de Desarrollo y Tecnogico, 1982. 169p.

McCUNE, B.; MEFFORD, M. J. PC-ORD: multivariate analysis of ecological data. Version 5. Oregon: USA, MjM Software, 2006. CD-ROM.

McCUNE, B.; GRACE, J. B. Analysis of ecological communities. Oregon: MJM Software, 2002. 300p. 
MORENO, J. A. Clima do Rio Grande do Sul. Porto Alegre: Secretaria da Agricultura, 1961. 73p.

MOSER, J. M. Solos. In: IBGE. Geografia do Brasil: Região Sul. Rio de Janeiro, 1990. p. 85-III.

NAPPO, M. E.; OLIVEIRA FILHO, A. T.; MARTINS, S. V. A estrutura do sub-bosque de povoamento homogêneos de Mimosa scabrella Bentham, em área minerada, em Poços de Caldas, MG. Ciência Florestal, v. 10, n.2, p. 17-19, 2000.

NIMER, E. Clima. In: IBGE. Geografia do Brasil: Região Sul. Rio de Janeiro, 1990. p.5I-I87.

ODUM, E. P. Ecologia. 2. ed. Rio de Janeiro: Guanabara Koogan, 1988. 434p.

PALMER, M. W. Putting things in even better order: the advantages of canonical correspondence analysis. Ecology, v. 74, p. 22I5-2230, 1993.

PILLAR, V. P.; MÜLLER, S. C.; CASTILHOS, Z. M. S.; JACQUES, A. V. A. Campos Sulinos - conservação e uso sustentável da biodiversidade. Brasília: Ministério do Meio Ambiente, 2009. 409p.

REICHARDT, K.; TIMM, L. C. Solo, Planta e Atmosfera. Conceitos, Processos e Aplicações. São Paulo: Manole, 2004. 478p.

RORATO, D. G. Fitossociologia de espécies nativas de mata ciliar no entorno do Reservatório Divisa, São Francisco de Paula, RS. 20 I2. I09f. Dissertação (Mestrado em Engenharia Florestal) - Universidade Federal de Santa Maria, Santa Maria, 2012.
SANTOS, R. M. et al. Identity and relationships of the Arboreal Caatinga among other floristic units of seasonally dry tropical forests (SDTFs) of north-eastern and Central Brazil. Ecology and Evolution, v. 2, n. 2, p. 409-428, 2012.

SBCS/CQFS - SOCIEDADE BRASILEIRA DE CIÊNCIA DO SOLO. Manual de adubação e calagem para os Estados do Rio Grande do Sul e Santa Catarina. 10. ed. Porto Alegre: Comissão de Química e Fertilidade do Solo, 2004. 400p.

SILVA, L. S.; CAMARGO, F. A. O; CERETTA, C. A. Composição da fase sólida orgânica do solo. In: MEURER, E.J. Fundamentos de química do solo. 3 ed. Porto Alegre: Evangraf, 2006. Cap.3, p. 63-90.

SILVA, R. F.; LUPATINI, M. L.; ANTONIOLLI, Z. A.; LEAL, L. T.; JUNIOR, C. A. M. Comportamento de Peltophorum dubium (Spreng.) Taub., Paraptadenia rigida (Benth.) Brenan e Enterolobium contortisiliquum (Vell.) Morong cultivadas em solo contaminado com cobre. Ciência Florestal, v. 21, n. I, p. I03-II0, 201 I.

STRECK, E. V. et al. Solos do Rio Grande do Sul. 2. ed. Porto Alegre: EMATER/RS-ASCAR, 2008. 222p.

TEDESCO, M.J.; GIANELLO, C.; BISSANI, C. A.; BOHNEN, H.; VOLKWEISS, S. J. Análises de solos, plantas e outros materiais. 2. ed. Porto Alegre: UFRGS, 1995. I74p.

TONETTO, T. S. Variabilidade de solos em área de tensão ecológica entre campo e Floresta Ombrófila Mista na Barragem do Divisa - São Francisco de Paula/RS. 65f. (Relatório de Estágio)-Universidade Federal de Santa Maria, Santa Maria, 201 I.

YRUELA, I. Copper in plants: acquisition, transport and interactions. Functional Plant Biology, v. 36, n.5, p. 409-430, 2009. 
